\title{
Simulation of Sequencing Rules Using Witness in a Milling Job Shop
}

\author{
Liqi Ang ${ }^{1}$, Kuan Yew Wong ${ }^{1}$ and Wai Peng Wong ${ }^{2}$ \\ ${ }^{1}$ Faculty of Mechanical Engineering, Universiti Teknologi Malaysia, UTM Skudai, Malaysia. \\ ${ }^{2}$ School of Management, Universiti Sains Malaysia, Penang, Malaysia.
}

\begin{abstract}
Simulation is essential when studying manufacturing processes or designing production systems. This project was a real case study which involved a job shop with five similar CNC milling machines. A total of six jobs were performed and each of them consisted of a different set of operations. The sequence of the six jobs to enter the system was determined by the sequencing rules including shortest setup time (SST), shortest processing time (SPT), shortest processing and setup time (SPST), earliest due date (EDD), least process (LP), and lowest volume (LV). The setup time was taken into consideration to make the results more realistic. Due to the complexity of the model, WITNESS was used to simulate all the sequencing rules. The best approach was then determined by comparing the results of each rule. By doing this, the case company would be able to make a better decision on which job should be processed first instead of selecting it randomly among the jobs.
\end{abstract}

Keywords: Sequencing Rules; WITNESS; Simulation; Job Shop; CNC Milling Machines

\section{Introduction}

The objectives of this study are to apply various sequencing rules in a company with a high-mix-low-volume manufacturing environment and to simulate all the sequencing rules in order to suggest the best approach for manufacturing multiple high-mix-low-volume products. Proper scheduling leads to increased efficiency and capacity utilization, reduced time required to complete tasks, and consequently increased profitability of an organization (Vinod and Sridharan, 2008). The most common high-mix-low-volume environment is in a job shop. This project is a simulation-based experimental study of sequencing rules for scheduling a dynamic job shop with five similar CNC milling machines.

The job-shop scheduling problem (JSSP) is one of the most critical problems in scheduling (Jayamohan and Rajendran,
2004). It aims to allocate a number of machines over time to perform a set of jobs with certain constraint conditions in order to optimize a certain criterion for example, minimizing the make-span (Yang et al., 2010). In the classical job shop scheduling problem, jobs are processed to completion on machines. Each job has distinct routes according to the technology constraints which are fixed and known. Every machine requires a setup period before any operation. Setup time is defined as the time interval between the end of processing of the current job and the beginning of processing of the next job (Vinod and Sridharan, 2008). Setup is the activities to prepare a machine or workstation to perform the next operation. For example, setting up a CNC milling machine includes keying in all the commands, cleaning up the coolant used for the previous operation, inserting the cutting tool or jig, and getting the zero point. Over the years, many sequencing rules have been proposed by 
many researchers but no single rule has been found to perform well for all important criteria such as mean flow time, mean tardiness and utilization. The choice of a sequencing rule depends on which criterion is intended to be improved upon (Holthaus and Rajendran, 1997).

Traditionally, when dealing with scheduling problems, researchers tend to neglect setup time. By doing this, the complexity of the scheduling problem is reduced but the result may be unrealistic. In this study, setup time is taken into account when applying all the sequencing rules. There are six sequencing rules selected in this study which are shortest setup time (SST), shortest processing time (SPT), shortest processing and setup time (SPST), earliest due date (EDD), least process (LP), and lowest volume (LV) (Holthaus and Rajendran, 1997; Rajendran and Holthaus, 1999). The performance of each rule can be measured by determining the average work in progress (WIP), total completion time, etc. WITNESS is used to simulate all the six sequencing rules proposed in this study. By comparing the results obtained, the best rule can be identified.

\section{Job Shop Model}

The job shop consists of five CNC milling machines $\{\mathrm{m}=1,2,3,4,5\}$. Each machine is similar and is multi-purposed. This means that each machine can perform all processes for all jobs by changing its setup. The job shop performs in a make-to-order style and the production is according to customer orders. Six jobs arrive at the same time $\{i=1,2,3,4,5,6\}$ and all of them have different due dates. Each of the jobs has a specific set of operations that needs to be performed in a specified sequence. The maximum number of processes that will be performed on a job is $6\{j=1,2,3,4,5,6\}$. Processing time for all the jobs in any of the machines is stochastic. Each process requires a different setup time on a machine. The jobs will just enter any of the machines which are vacant at that particular time and they do not necessarily start with process 1 at machine 1. Due to this, the flow paths for all the jobs through the 5 similar CNC milling machines are very complicated.

\section{Priority Selection}

The terminologies and sequencing rules adopted in this study are described in Tables 1 and 2 respectively.

Table1: Terminologies

\begin{tabular}{|c|l|}
\hline Symbol & Description \\
\hline $\mathbf{m}$ & $\begin{array}{l}\text { Index of the machine for which the job to be processed } \\
\text { next has to be selected }\end{array}$ \\
\hline $\mathbf{t}$ & Time at which the priority values are calculated \\
\hline $\mathbf{i}$ & Index of the job for which the priority values are calculated \\
\hline $\mathbf{j}$ & Index of the operation of job i \\
\hline $\boldsymbol{d}_{\boldsymbol{i}}$ & Due date of job i \\
\hline $\boldsymbol{s}_{i j}^{m}$ & Setup time of operation j of job i on machine m \\
\hline $\boldsymbol{p}_{i j}^{\boldsymbol{m}}$ & Processing time of operation j of job i on machine m \\
\hline $\boldsymbol{Q}_{\boldsymbol{i}}$ & Volume of order for job i \\
\hline $\boldsymbol{p}_{\boldsymbol{i}}$ & Number of operations for job i \\
\hline $\boldsymbol{V}_{\boldsymbol{i}}^{t}$ & Priority value of job i at time t \\
\hline
\end{tabular}


Table 2: Rules and Sequence Selection

\begin{tabular}{|c|c|c|}
\hline Rules & Formula & Selection \\
\hline Shortest setup time (SST) & $V_{i}^{\mathrm{z}}=s_{i \mathrm{i}}^{\mathrm{m}}$ & $\begin{array}{l}\text { Highest priority is given to job i with } \\
\text { minimum } V_{i}^{t} \text { value. }\end{array}$ \\
\hline $\begin{array}{l}\text { Shortest processing } \\
\text { time (SPT) }\end{array}$ & $V_{\mathrm{i}}^{\mathrm{z}}=\mathrm{p}_{\mathrm{ij}}^{\mathrm{m}}$ & $\begin{array}{l}\text { Highest priority is given to job i with } \\
\text { minimum } V_{i}^{t} \text { value. }\end{array}$ \\
\hline $\begin{array}{l}\text { Shortest processing and } \\
\text { setup time (SPST) }\end{array}$ & $V_{i}^{t}=p_{i j}^{m}+s_{i j}^{m}$ & $\begin{array}{l}\text { Highest priority is given to job i with } \\
\text { minimum } V_{i}^{t} \text { value. }\end{array}$ \\
\hline Earliest due date (EDD) & $V_{i}^{t}=d_{i}$ & $\begin{array}{l}\text { Highest priority is given to job i with } \\
\text { minimum } V_{i}^{t} \text { value. When the due } \\
\text { date is same, select SPST. }\end{array}$ \\
\hline Least process (LP) & $V_{i}^{t}=p_{i}$ & $\begin{array}{l}\text { Highest priority is given to job i with } \\
\text { minimum } V_{i}^{t} \text { value. When the } \\
\text { number of operations is same, select } \\
\text { SPST. }\end{array}$ \\
\hline Lowest volume (LV) & $V_{i}^{t}=Q_{i}$ & $\begin{array}{l}\text { Highest priority is given to job i with } \\
\text { minimum } V_{i}^{t} \text { value. When the lot } \\
\text { size is same, select SPST. }\end{array}$ \\
\hline
\end{tabular}

\section{Simulation Modelling}

Due to the complexity of this five-similarmachine job shop model, it is very difficult to measure the performance of each sequencing rule. As a result, WITNESS (Markt and Mayer, 1997; Olugu and Wong, 2008) was used to simulate all the rules in order to determine the best approach. Before building the simulation model, the following data were first collected and their probability distributions were determined using the goodness-of-fit test.

1) Cycle time \& setup time for every process of each of the 6 jobs.

2) Inspection time \& packaging time for the final process of each job.

3) Due date \& lot size for each job.

Several assumptions were also made, which include:

1) Each machine can perform only one operation at a time on any job.
2) Once an operation has begun on a machine, it must not be interrupted.

3) An operation of a job cannot be performed until its preceding operations are completed.

4) There is no alternate routing. The jobs must undergo every process in sequence.

5) Operation processing time and number of operable machines are known in advance.

6) There is a single unlimited queuing space for all the machines.

7) Each machine is continuously available for production; no breakdown time is considered.

8) All the parts are perfectly processed; no scrap or rework is considered.

9) All the jobs with specific lot sizes are available when the system starts. 


\section{Experimentation}

This simulation model can be considered as a terminating system because it runs and stops after a particular period. A terminating system does not require a warm-up period. This system has a fixed starting condition and a naturally occurring event (event $E$ ) that marks the end of the system (Banks et al., 2005). The naturally occurring event in this case is when all the operations are completed for all the parts at $T_{E}$. Hence, $T_{E}$ is the total completion time of all the jobs and for each sequencing rule applied, $T_{E}$ has a different value. The experiment was run for 3 times and each replication used a different set of random numbers. There was no specified length of simulation; the system ran continuously until all the parts were completely processed and shipped. The execution mode was set as restart mode.

\section{Verification and Validation}

In order to verify the model, the codes and all the input and output rules used in WITNESS were checked by experts. The flow and action of the simulation model were tested. The step button in the run toolbar in WITNESS was used to examine the execution of the model step by step. It was observed that all the parts followed their specified routings throughout the system and the sequence of the parts entering the system was as desired.

To validate the model, the data from the actual production system were compared with the results obtained from simulation. In the actual job shop production system, 6 working days ( 8 hours x 60 minutes x 6 days $=2880$ minutes) were approximately needed to complete all the 6 jobs. With manual estimation, when adding up the cycle time, setup time, inspection time and packaging time for all the jobs with specified lot sizes, the total time was around 3000 minutes. Based on the results obtained from WITNESS, the total completion time of all the jobs varied from 2835 to 2875 minutes. Therefore, the simulation model built in this study (as shown in Figure 1) could be considered as valid because the results obtained were close to the actual production system's data.



Figure 1: Simulation Model 


\section{Results and Discussions}

The performance of the six sequencing rules was measured in two aspects which were particularly emphasized by the case company; they were average work in progress (WIP) and average total completion time. For each of these measures, the average value of three replications was calculated and the results are discussed in the ensuing paragraphs.

\section{Average Work in Progress (WIP)}

Any industry will aim to reduce work in progress (WIP) as it can reduce inventory waste. Figure 2 shows that the SPST rule emerges as the best in minimizing the average WIP in the system. This is because a job with the shortest processing time and setup time is selected first and it stays in the system in a relatively short time. Hence, it is believed that the average WIP will be the lowest. The second best rule in this aspect is SPT, followed by SST, LP and LV. The time oriented rules, i.e. SPST, SPT and SST occupy the top three and thus, it can be concluded that low processing time and setup time play an important role in minimizing WIP. The worst rule in this aspect is EDD because it concentrates only on due date.

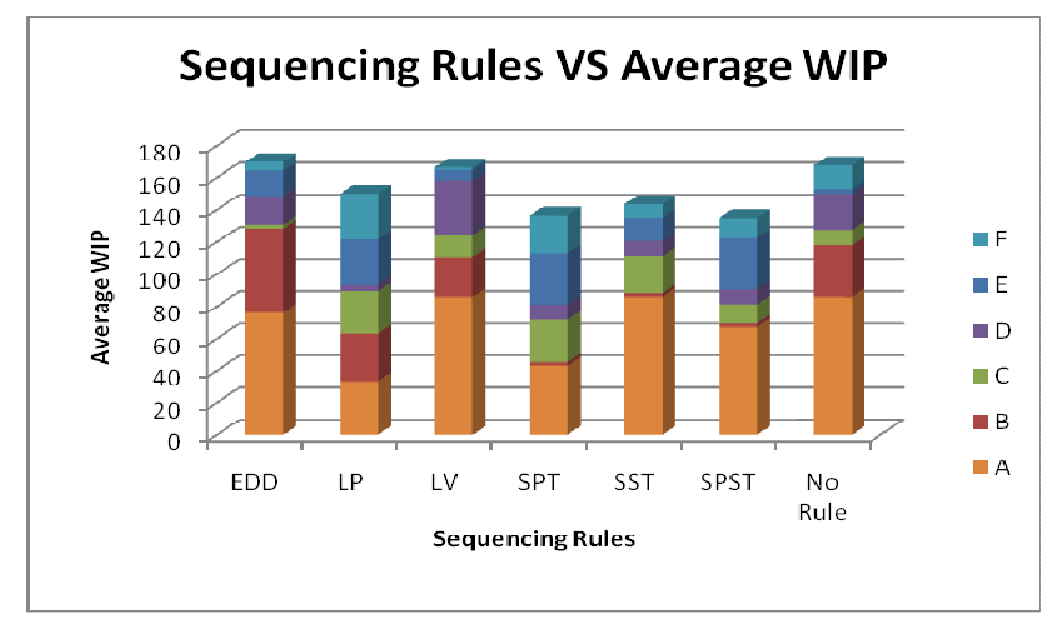

Figure 2: Average Work in Progress for Different Sequencing Rules

\section{Average Total Completion Time}

The total completion time was obtained by running the simulation model until there was no event scheduled. The time when all the parts in the system have been shipped was considered as the total completion time. Figure 3 shows that the SPST rule emerges as the best in minimizing the average total completion time. The time oriented rule - SPST proves significant to reduce the make-span. The second best rule in this aspect is $\mathrm{LV}$, followed by SST, SPT and EDD. The worst rule in this aspect is LP because it does not take into account the cycle time and setup time, but considers only the number of operations. 


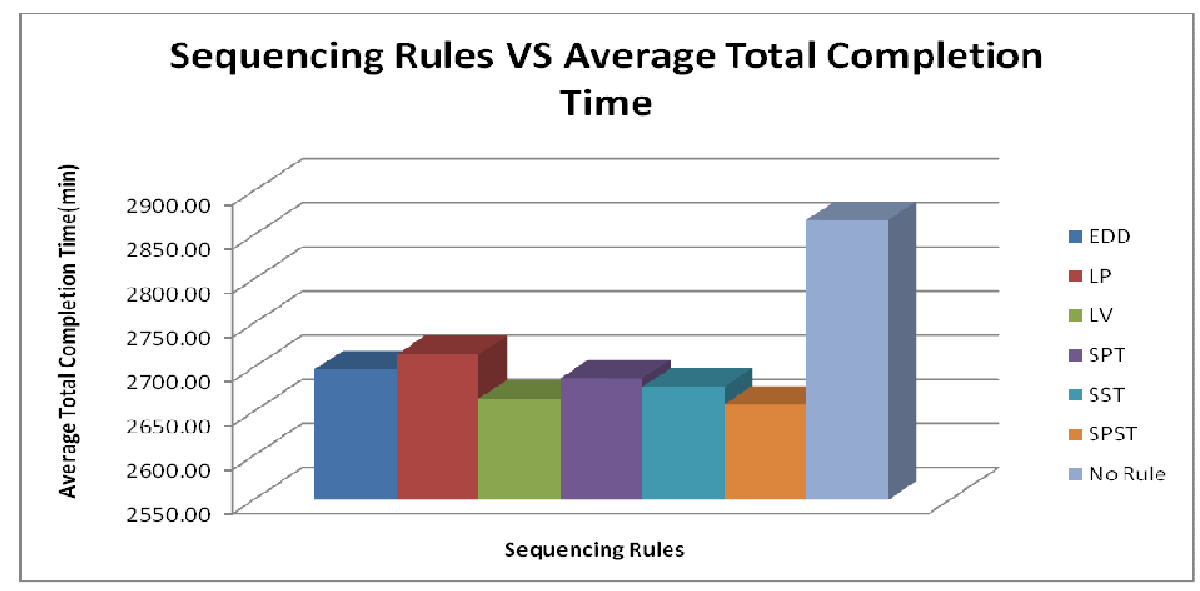

Figure 3: Average Total Completion Time for Different Sequencing Rules

\section{Conclusions}

This study focused on suggesting the best sequencing rule for a job shop with five similar CNC milling machines. Due to the complexity of the model, WITNESS was used to simulate the model and to obtain the results. By comparing the performance of each rule in terms of average WIP and average total completion time, the best rule for each aspect could be determined. In general, SPST is the best option to minimize the average WIP and average total completion time in the system. Nevertheless, it should be highlighted that no single rule is effective in optimizing all types of performance measures. Different situations will require different sequencing rules to meet a company's requirement.

\section{References}

Banks, J., Carson, J. S., Nelson, B. L. \& Nicol, D. M. (2005). 'Discrete-Event System Simulation,' Pearson Prentice Hall, New Jersey.

Holthaus, O. \& Rajendran, C. (1997). "Efficient Dispatching Rules for Scheduling in a Job Shop," International Journal of Production Economics, 48 (1). 87-105.

Jayamohan, M. S. \& Rajendran, C. (2004). "Development and Analysis of Cost-Based Dispatching Rules for Job Shop Scheduling," European Journal of Operational Research, 157 (2). 307-321.
Markt, P. L. \& Mayer, M. H. (1997). "Witness Simulation Software: A Flexible Suite of Simulation Tools," Proceedings of the 1997 Winter Simulation Conference, Atlanta, Georgia, 711-717.

Olugu, E.U. \& Wong, K.Y. (2008). "Simulation Study on Lens Manufacturing Process Flow," Proceedings of the Second Asia International Conference on Modelling and Simulation, Kuala Lumpur, Malaysia, 806-811.

Rajendran, C. \& Holthaus, O. (1999). “A Comparative Study of Dispatching Rules in Dynamic Flowshops and Jobshops," European Journal of Operational Research, 116 (1). 156-170.

Vinod, V. \& Sridharan, R. (2008). "Scheduling a Dynamic Job Shop Production System with SequenceDependent Setups: An Experimental Study," Robotics and Computer-Integrated Manufacturing, 24 (3). 435-449.

Yang, S., Wang, D., Chai, T. \& Kendall, G. (2010). "An Improved Constraint Satisfaction Adaptive Neural Network for Job-Shop Scheduling," Journal of Scheduling, 13 (1). 17-38. 